\title{
ON ISOMORPHIC GROUPS AND HOMEOMORPHIC SPACES
}

\section{J. S. YANG}

\begin{abstract}
Let $C(X, G)$ denote the group of continuous functions from a topological space $X$ into a topological group $G$ with the pointwise multiplication. Some classes of $S Q$-pairs and properties of the corresponding topological group $C(X, G)$ with the compact-open topology are investigated. We also show that the existence of a group isomorphism between groups $C(X, G)$ and $C(Y, G)$ implies the existence of a homeomorphism between $X$ and $Y$, if $(X, G)$ and $(Y, G)$ are $S Q$-pairs.
\end{abstract}

1. Introduction. For a topological space $X$ and a topological group $G$, let $C(X, G)$ be the group of all continuous functions from $X$ into $G$ with the pointwise multiplication, that is, $(f g)(x)=f(x) g(x)$; the identity element of the group $C(X, G)$ is the constant function $I_{0}(X, G)$, or simply $I_{0}$, which maps every $x$ in $X$ into the identity element $e$ of $G$. It is well known that if $C(X, G)$ is endowed with the compact-open topology, it becomes a topological group. It is clear that if $h$ is a homeomorphism of $X$ onto $Y$, then $f \rightarrow f \circ h$ is an isomorphism from $C(Y, G)$ onto $C(X, G)$ which maps every constant function on $Y$ into the corresponding constant function on $X$. We are concerned, in this paper, with the question: If a group isomorphism exists between $C(Y, G)$ and $C(X, G)$ which maps every constant function on $Y$ into the corresponding constant function on $X$, does there exist a homeomorphism between $X$ and $Y$ ? In general, the answer to this question is, of course, no, for we may take $X$ to be a noncompact pseudocompact space, and then there is a ring isomorphism between the rings $C(X, R)$ and $C(\beta X, R)$ but $X$ and $\beta X$ are not homeomorphic.

We find that the answer to the above question is yes for certain pairs $(X, G)$ of topological space $X$ and topological group $G$. Such pairs are termed $S Q$-pairs as defined in [9]. $\S 3$ is devoted to proving this assertion by showing first that, if $X$ is a $k$-space, $X$ is homeomorphic to the space of all $c$-continuous homomorphisms of the topological group $C(X, G)$ onto the topological group $G$ with $F$-normal subgroups as kernels and

Presented to the Society, November 24, 1972; received by the editors February 22, 1973.

AMS (MOS) subject classifications (1970). Primary 54C35, 22 A05.

Key words and phrases. Topological group, pointwise multiplication, group, isomorphism, homomorphism, $k$-space, compact-open topology, jointly continuous.

(c) American Mathematical Society 1974 
endowed with the compact-open topology. We disclose some classes of $S Q$-pairs, and some properties of $C(X, G)$ in $\$ 2$.

All topological spaces considered here are assumed to be Hausdorff.

2. $S Q$-pairs. For each $p$ in $X$, let $M_{p}=\{f \in C(X, G): f(p)=e\}$, and let $h_{p}$ be the evaluation map of $C(X, G)$ onto $G$ defined by $h_{p}(f)=f(p)$. For each $r$ in $G$, let $r$ denote the constant function in $C(X, G)$ which maps $X$ into $r$. Then $h_{p}$ is a continuous homomorphism of $C(X, G)$ onto $G$ with $M_{p}$ as its kernel and maps every constant function $r$ into $r$. Hence we see that $C(X, G) / M_{p}$ is isomorphic to $G$ under the continuous isomorphism that maps every coset $c M_{p}$ into $c$. Note that for each $p$ in $X$, every coset $c M_{p}$ contains exactly one constant map, namely $c$. For the sake of convenience, let us call a homomorphism of $C(X, G)$ (or $C(X, G) / M$ ) onto $G$ a $c$-homomorphism if it maps every $\boldsymbol{r}$ (resp. $\boldsymbol{r} M$ ) into $r$. Every evaluation map is a $c$-continuous homomorphism of $C(X, G)$ onto $G$.

In contrast to the fact that every nonzero homomorphism of $C(X)=$ $C(X, R)$ onto $R$ is a $c$-homomorphism $[5,10.5]$, not every continuous homomorphism of $C(X, G)$ onto $G$ is a $c$-continuous homomorphism, as the following example shows.

EXAMPLE. Let $G$ be the additive group of integers modulo 2 with the discrete topology. Then $C(G, G)=\left\{I_{0}, f_{1}, f_{2}, f_{3}\right\}$, where $f_{1}$ is the function which maps $G$ into $1, f_{2}$ is the function which maps 1 into 1 and 0 into 0 , and $f_{3}$ is the one which maps 0 into 1 and 1 into 0 . The compact-open topology for $C(G, G)$ is the discrete topology. If we define a mapping $h$ : $C(G, G) \rightarrow G$ by defining $h\left(I_{0}\right)=h\left(f_{1}\right)=0, h\left(f_{2}\right)=h\left(f_{3}\right)=1$, then $h$ is an onto homomorphism, yet it is not a $c$-homomorphism.

For $f \in C(X, G)$, we let $Z(f)=\{x \in X: f(x)=e\}$, and for a subgroup $M$ of $C(X, G)$, let $Z(M)=\{Z(f): f \in M\}$. Note that, for any $f$ and $g$ in $C(X, G)$,

$$
Z(f g) \supset Z(f) \cap Z(g), \quad Z\left(f^{-1}\right)=Z(f) \quad \text { and } \quad Z\left(f g f^{-1}\right)=Z(g) .
$$

Definition 1 [9]. We shall call a pair $(X, G)$ of a topological space $X$ and a topological group $G$ an $S$-pair if, for each closed subset $C$ of $X$ and $x \notin C$, there exists $f \in C(X, G)$ such that $Z(f) \supset C$ and $f(x) \neq e$.

It is clear that $(X, R)$ is an $S$-pair for every completely regular space, and that if $(X, G)$ is an $S$-pair then $X$ is completely regular.

REMARK 1. If $X$ is a topological space such that each $x$ in $X$ has a local base $U_{x}$ satisfying the property that, for each $U$ in $U_{x}$ there exists a continuous function $f$ of $\bar{U}$ into $G$ such that $f(x) \neq e$ but $f(y)=e$ for each $y$ in $\bar{U}-U$, then $(X, G)$ is an $S$-pair. To see this, let $C$ be a closed subset of $X$ and $x \notin C$. Then, for some $U$ in $U_{x}, x \in U \subset X-C$; and let $f$ be a continuous function on $\bar{U}$ into $G$ such that $f(x) \neq e$ but $f(y)=e$ for each $y$ 
in $\bar{U}-U$. Define $g: X \rightarrow G$ such that $g=f$ on $\bar{U}$ and $g(y)=e$ for $y \notin \bar{U}$. Then $g \in C(X, G), Z(g) \supset C$, and $g(x) \neq e$.

REMARK 2. If $X$ is completely regular, and $G$ is path connected, then $(X, G)$ is an $S$-pair. To see this let $t \neq e$ be in $G$. If $C$ is a closed subset of $X$ and $x \notin C$, let $f$ be a continuous function of $X$ into $[0,1]$ such that $f(x)=1$ and $f(C)=\{0\}$, and let $g:[0,1] \rightarrow G$ be the path such that $g(0)=e$ and $g(1)=t$. Then $g \circ f$ is the desired function in $C(X, G)$.

RemarK 3. For every zero-dimensional space $X,(X, G)$ is an $S$-pair.

We point out that, if $B$ is a closed subset of $X$ and $(X, G)$ is an $S$-pair, then $(B, G)$ is also an $S$-pair.

Definition 2 [9]. (1) A normal subgroup $M$ of $C(X, G)$ is called an $F$-normal subgroup if $\{Z(f): f \in M\}$ has the finite intersection property.

(2) A pair $(X, G)$ of a topological space $X$ and a topological group $G$ is called a $Q$-pair if whenever $M$ is an $F$-normal subgroup of $C(X, G)$ such that $C(X, G) / M$ is isomorphic to $G$ by a $c$-isomorphism, then $\cap Z(M) \neq \varnothing$.

It is clear that if $X$ is a completely regular space such that $(X, R)$ is a $Q$-pair, then $X$ is realcompact. As pointed out in [9], $(X, G)$ is a $Q$-pair if $X$ can be embedded into $G$ as a subspace of $G$. Since every completely regular space $X$ is a closed subspace of the free topological group $F(X)$ generated by $X$, and every topological group can be embedded as a closed subgroup of a path connected and locally path connected topological group [6], we see that for every completely regular space $X$ there exists a path connected and locally path connected topological group $G$ such that $(X, G)$ is an $S Q$-pair. If $X$ is compact, $(X, R)$ is an $S Q$-pair.

If $(X, G)$ is a $Q$-pair, then the only $F$-normal subgroups of $C(X, G)$ such that $C(X, G) / M$ is $c$-isomorphic to $G$ are of the form $M_{p}, p \in X$ [9]. Thus we have the following:

Proposition 4. An S-pair $(X, G)$ is a Q-pair if and only if every c-homomorphism $h$ of $C(X, G)$ onto $G$ with an $F$-normal subgroup as its kernel is of the form $h_{p}$ for some $p \in X$.

Proof. For the necessity, let $M$ be the kernel of $h$, then $C(X, G) / M$ is $c$-isomorphic to $G$. Hence there is $p \in \cap Z(M)$ such that $M=M_{p}$. Therefore ker $h=\operatorname{ker} h_{p}$. Now for $f \in C(X, G)$, let $f(p)=c$, and let $g=f c^{-1}$, then $g \in M_{p}=M$. Hence

$$
h(f)=h(g c)=h(g) h(c)=h(g) c=c=f(p)=h_{p}(f) .
$$

This shows that $h=h_{p}$.

For the sufficiency, suppose $M$ is an $F$-normal subgroup of $C(X, G)$ such that $C(X, G) / M$ is $c$-isomorphic to $G$ by the $c$-isomorphism $k$. Let $h=k \circ \alpha$, where $\alpha$ is the natural map of $C(X, G)$ onto $C(X, G) / M$. Then $h$ 
is a $c$-homomorphism of $C(X, G)$ onto $G$ with $M$ as its kernel. Hence there is a unique $p \in X$ such that $h=h_{p}$, and thus $M=M_{p}$.

Following [7], we call a topological space $X$ a $V$-space if for points $p, q, x$, and $y$ of $X$, where $p \neq q$, there exists a continuous function $f$ of $X$ into itself such that $f(p)=x$ and $f(q)=y$. It is shown in [7] that every completely regular path connected space and every zero-dimensional space is a $V$-space.

Recall that a topological space $X$ is said to be an $S$-space if, for each pair of distinct points of $X$, there is a continuous real-valued function on $X$ whose values at these points do not coincide. R. Arens defined it in [1], and has shown that, if the space $C(X, R)$ satisfies the first axiom of countability and $X$ is an $S$-space, then $X$ is hemicompact. Adopting the same line of argument, we have the following:

Theorem 5. If $(X, G)$ is an S-pair, $G$ is a V-space, and if $C(X, G)$ satisfies the first axiom of countability, then $X$ is hemicompact and $G$ is metrizable.

Proof. Since $G$ can be embedded as a retract of $C(X, G), G$ is metrizable. For the hemicompactness of $X$, the proof is not different from that of [1, Theorem 8] and thus omitted.

It is remarked that, if $X=\bigcup_{n=1}^{\infty} C_{n}$ where $C_{1} \subset C_{2} \subset C_{3}, \cdots$, is hemicompact and if $\left\{V_{n}\right\}$ is a countable local base for $e$ in $G$, then $\left\{\left(C_{n}, V_{m}\right)\right\}$ is a local base at $I_{0}$ in $C(X, G)$, and hence $C(X, G)$ is metrizable, where $\left(C_{n}, V_{m}\right)=\left\{f \in C(X, G): f\left(C_{n}\right) \subset V_{m}\right\}$.

Lemma 6. Let $(X, G)$ be an $S$-pair, and let $\Omega$ be an open covering for $X$. For each closed subset $C$ of $X$ contained in some member of $\Omega$ and for each open neighborhood $U$ of $e$ in $G$, let $(C, U)=\{f \in C(X, G): f(C) \subset U\}$. Then the topology $t$ for the group $C(X, G)$ having the family of sets of the form $(C, U)$ as subbasic neighborhoods of $I_{0}$ is jointly continuous, that is, the map $P: X \times C(X, G) \rightarrow G$ defined by $P(f, x)=f(x)$ is continuous.

Proof. Let $f \in C(X, G), x \in X$, and let $W$ be a neighborhood of $f(x)$. Then $f(x) U \subset W$ for some open set $U$ in $G$ containing $e$, and hence $x \in f^{-1}(f(x) V) \cap O$, where $x \in O \in \Omega$ and $V$ an open neighborhood of $e$ such that $V^{2} \subset U$. If $C$ is a closed neighborhood of $x$ such that $C \subset f^{-1}(f(x) V) \cap O$, then, for $g \in f(C, V)$ and $y \in C, g(y) \in f(y) V \subset$ $f(x) U \subset W$. Hence $P$ is continuous.

THEOREM 7. Let $(X, G)$ be an $S$-pair, where $G$ is a $V$-space. If there exists a smallest jointly continuous topology $t$ for the group $C(X, G)$, then $X$ is locally compact. 
Proof. The proof is similar to that of [1, Theorem 3]. Let $a$ be an element of $G$ different from $e$, and let $U$ be a neighborhood of $e$ in $G$ such that $a \notin U$, and let $x \in X$. By the joint continuity of $t$, let $V$ be a neighborhood of $x$, and $W$ a $t$-neighborhood of $I_{0}$ such that $g(V) \subset U$ for every $g$ in $W$. We want to show that $\bar{V}$ is compact.

Let $\Omega$ be an open covering for $\bar{V}$, and let $\Omega^{\prime}=\{X-\bar{V}\} \cup \Omega$. Then $\Omega^{\prime}$ is an open covering for $X$. Let $t^{\prime}$ be the topology for $C(X, G)$ induced by $\Omega^{\prime}$ as described in Lemma 6 , then we have $t \subset t^{\prime}$. Hence there are closed sets $C_{i} \subset O_{i}$ of $X$ and open neighborhoods $U_{i}$ of $e$ in $G, i=1,2, \cdots, n$, such that $W^{\prime}=\bigcap_{i=1}^{n}\left(C_{i}, U_{i}\right)$ is contained in $W$. Let $O=V-\bigcup_{i=1}^{n} C_{i}$, and suppose that $p \in O$. Then there is $f$ in $C(X, G)$ such that $Z(f) \supset X-O$ and $f(p) \neq e$. Let $g$ be a continuous function of $G$ into itself with $g(e)=e$ and $g(f(p))=a$, and let $g=g \circ f$. Then $h(X-O)=e$ and $h(p)=a$, hence $h \in W^{\prime}$. But $p$ is in $V$ and $h(p)=a \notin U$; we have $h \notin W$ which is impossible. Hence $O=\varnothing$, and we have $\bar{V} \subset \bigcup_{i=1}^{n} C_{i} \subset \bigcup_{i=1}^{n} O_{i}$. Therefore $\bar{V}$ is compact.

Corollary. If $(X, G)$ is an S-pair, where $G$ is a $V$-space, and $X \times$ $C(X, G)$ is a k-space, where $C(X, G)$ has the compact-open topology, then $X$ is locally compact.

Proof. If $X \times C(X, G)$ is a $k$-space, then the compact-open topology for $C(X, G)$ is jointly continuous [2]; hence $X$ is locally compact.

The above corollary generalizes a result in [2]. As an application, we show in the following example that the product of two topological groups which are $k$-spaces need not be a $k$-space, a fact pointed out by N. Noble [8].

ExAMPLE. Let $X$ be the dual space of an infinite-dimensional Fréchet space with the compact-open topology. Then $X$ is a topological group which is a hemicompact $k$-space but is not locally compact. If $G$ is any metrizable topological group which is also a $V$-space such that $(X, G)$ is an $S$-pair, then $C(X, G)$ is metrizable by the remark following Theorem 5. Since $X$ is not locally compact, $X \times C(X, G)$ is a topological group but is not a $k$-space as follows from the above corollary. This example was cited by N. Noble [8] for the case where $G$ is the additive group of real numbers.

3. Isomorphic groups. This section is devoted to prove the following:

THEOREM 8. Suppose that $(X, G)$ and $(Y, G)$ are SQ-pairs. If there exists an isomorphism between groups $C(Y, G)$ and $C(X, G)$ which maps every constant function on $Y$ into the corresponding constant function on $X$, then $X$ and $Y$ are homeomorphic.

All pairs $(Z, G)$ considered in this section are assumed to be $S Q$-pairs. Since every noncompact pseudocompact space $X$ is not realcompact, 
$(X, R)$ cannot be a $Q$-pair, thus Theorem 8 is false if $(X, G)$ is not a $Q$-pair.

In order to establish Theorem 8 , we first prove that, if $X$ is a $k$-space, $X$ is homeomorphic to the space of all $c$-continuous homomorphisms of the topological group $C(X, G)$ onto the topological group $G$ with $F$ normal subgroups as kernels and endowed with the compact-open topology; let $H(X, G)$ denote such a space of $c$-continuous homomorphisms. For each $p \in X$, the evaluation map $h_{p}$ is in $H(X, G)$, hence the correspondence $p \rightarrow h_{p}$ defines a map $\mu$ from $X$ into $H(X, G)$.

THEOREM 9. If $X$ is a $k$-space, the mapping $\mu$ is a homeomorphism of $X$ onto $H(X, G)$.

Proof. Proposition 4 implies that $\mu$ is onto.

If $p \neq q$ in $X$, there is $f \in C(X, G)$ such that $f(p) \neq f(q)$, hence $h_{p}(f) \neq$ $h_{q}(f)$. Thus $\mu$ is one-to-one.

The continuity of $\mu$ follows from Theorem 2 of [4], which states that if $F$ is a family of continuous functions from a $k$-space $X$ into a regular space $Y$ endowed with the compact-open topology, then the mapping $\theta: X \rightarrow C(F, Y)$ defined by $\theta(x)(f)=f(x)$ is continuous, where $C(F, Y)$ also has the compact-open topology.

It remains to show that $\mu$ is a closed map. Let $C$ be a closed subset of $X$. Then $\mu(C)=\left\{h_{x}: x \in C\right\}$. Let $\left\{h_{x_{n}}\right\}_{n \in A}$ be a net in $\mu(C)$ such that $h_{x_{n}} \rightarrow h_{x}$ in $H(X, G)$, where $x_{n} \in C$ for each $n \in A$. If $x \notin C$, then there exists an $f$ in $C(X, G)$ such that $f(x) \notin \operatorname{cl}[f(C)]$. But $h_{x_{n}}(f) \rightarrow h_{x}(f)$ in $G$; we have $f\left(x_{n}\right) \rightarrow f(x)$ in $G$, hence $f(x) \in \operatorname{cl}[f(C)]$, a contradiction. Hence $x \in C$ and $\mu(C)$ is closed.

REMARK 10. The hypothesis that $X$ is a $k$-space in Theorem 9 is merely to assure the continuity of $\mu$. In fact, if $H(X, G)$ is given the pointopen topology instead of the compact-open topology, the mapping $\mu$ is easily seen to be continuous without assuming that $X$ is a $k$-space.

Suppose now that $\theta: X \rightarrow Y$ is a continuous map of a $k$-space $X$ into a $k$-space $Y$. Define $\theta^{\prime}: C(Y, G) \rightarrow C(X, G)$ by setting $\theta^{\prime}(g)=g \circ \theta$ for each $g$ in $C(Y, G)$ into the corresponding constant function in $C(X, G)$. Note that if $h_{x} \in H(X, G)$, then $h_{x} \circ \theta^{\prime}$ is in $H(Y, G)$. Hence we have a continuous mapping $\theta^{\prime \prime}$ of $H(X, G)$ onto $H(Y, G)$ defined by $\theta^{\prime \prime}\left(h_{x}\right)=h_{x} \circ \theta^{\prime}$ for each $h_{x} \in H(X, G)$. It is easy to verify that the following diagram

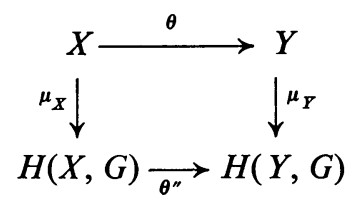

is commutative, where $\mu_{Z}: Z \rightarrow H(Z, G)$ is the mapping of Theorem 9 . 
THEOREM 11. Suppose that $X$ and $Y$ are $k$-spaces. Every continuous homomorphism $h: C(Y, G) \rightarrow C(X, G)$ which maps every constant function on $Y$ into the corresponding constant function on $X$, induces a unique continuous mapping $j$ of $X$ into $Y$ such that $j^{\prime}=h$. Furthermore, if $h$ is a topological isomorphism, then the induced mapping $j$ is a homeomorphism.

Proof. Let $h^{\prime}$ be the mapping of $H(X, G)$ into $H(Y, G)$ defined by $h^{\prime}\left(h_{x}\right)=h_{x} \circ h$ for each $h_{x} \in H(X, G)$. Since $X$ and $Y$ are $k$-spaces, $\mu_{X}$ and $\mu_{Y}$ are homeomorphisms by Theorem 9. If we define $j: X \rightarrow Y$ by setting $j=\mu_{Y}^{-1} \circ h^{\prime} \circ \mu_{X}$, then the above diagram shows that $j$ is continuous. Note that $j(x)=y$ if and only if $h(g)(x)=g(y)$ for each $g \in C(Y, G)$. If $j^{\prime}: C(Y, G) \rightarrow C(X, G)$ is the mapping defined by $j^{\prime}(g)=g \circ j$ for each $g \in C(Y, G)$, it is easy to verify that $j^{\prime}=h$.

If $r: X \rightarrow Y$ is any continuous mapping such that $r(x) \neq j(x)$ for some $x \in X$, then there exists an $f \in C(X, G)$ such that $f(r(x)) \neq f(j(x))$. Hence $r^{\prime} \neq j^{\prime}$, and the uniqueness of $j$ follows.

Now if $h$ is a topological isomorphism, then $j$ is onto and one-to-one (cf. [5, 10.2]), and $j^{-1}$ is continuous. Hence $j$ is a homeomorphism of $X$ onto $Y$, and the proof is completed.

As one may notice from the above proof, the introduction of the mapping $j$ depends solely on the homeomorphism of the maps $\mu_{X}$ and $\mu_{Y}$, and, as noted in Remark 10, the mapping $\mu$ is always a homeomorphism if $H(X, G)$ is endowed with the point-open topology which indeed coincides with the compact-open topology if the domain space is discrete [3]. With this remark, we can now prove Theorem 8 very easily; take discrete topologies for the groups $C(Y, G)$ and $C(X, G)$ then apply the proof of Theorem 11.

Remark 12. In fact, if we define an $S$-pair $(X, G)$ in a weaker form, (that is if we define $(X, G)$ to be an $S$-pair if, for each closed subset $C$ of $X$ and $x \notin C$ there exists an $f$ in $C(X, G)$ such that $f(x) \notin \operatorname{cl}[f(C)])$, then most of the results stated above, except perhaps Theorems 5 and 7 , hold.

\section{REFERENCES}

1. R. Arens, A topology for spaces of transformations, Ann. of Math. (2) 47 (1946), 480-495. MR 8, 165.

2. R. W. Bagley and J. S. Yang, On k-spaces and function spaces, Proc. Amer. Math. Soc. 17 (1966), 703-705. MR 33 \#693.

3. J. Dugundji, Topology, Allyn and Bacon, Boston, Mass., 1966. MR 33 \#1824.

4. D. Gale, Compact sets of functions and function rings, Proc. Amer. Math. Soc. 1 (1950), 303-308. MR 12, 119.

5. L. Gillman and M. Jerison, Rings of continuous functions, University Series in Higher Math., Van Nostrand, Princeton, N.J., 1960. MR 22 \#6994.

6. S. Hartman and J. Mycielski, On the imbeddings of topological groups into connected topological groups, Colloq. Math. 5 (1958), 167-169. MR 20 \#6480. 
7. K. D. Magill, Jr., Some homomorphism theorems for a class of semigroups, Proc. London Math. Soc. (3) 15 (1965), 517-526. MR 32 \#2499.

8. N. L. Noble, $k$-spaces and some generalizations, Doctoral Dissertation, University of Rochester, Rochester, N.Y., 1967.

9. J. S. Yang, Transformation groups of automorphisms of $C(X, G)$, Proc. Amer. Math. Soc. 39 (1973), 619-624.

Department of Mathematics, University of South Carolina, Columbia, South Carolina 29208 